\title{
Elevated beta-thromboglobulin and mean platelet volume levels may show persistent platelet activation in systemic lupus erythematosus patients
}

\author{
Seyit Uyar ${ }^{1, A, B, D-F}$, Gül Babacan Abanonu ${ }^{2, C, E}$, Seval Masatlıoğlu Pehlevan ${ }^{3, A, E}$, Cumali Karatoprak ${ }^{4, A, C}$, \\ Meral Uluköylü Mengüç一, ${ }^{2, B, C}$, Alper Daşkinn ${ }^{2,4, A, B}$, Süleyman Dolu ${ }^{1, A, C}$, Refik Demirtunç, ${ }^{2, A, E}$ \\ ${ }^{1}$ Department of Internal Medicine, University of Health Science, Antalya Training and Research Hospital, Turkey \\ 2 Department of Internal Medicine, University of Health Science, Haydarpaşa Numune Training and Research Hospital, Istanbul, Turkey \\ ${ }^{3}$ Department of Rheumatology, University of Health Science, Haydarpaşa Numune Training and Research Hospital, Istanbul, Turkey \\ ${ }^{4}$ Bezmialem Vakıf University Faculty of Medicine, Internal Medicine Clinic, Istanbul, Turkey \\ A - research concept and design; B - collection and/or assembly of data; C - data analysis and interpretation; \\ $D$ - writing the article; $E$ - critical revision of the article; $F$ - final approval of the article
}

\section{Address for correspondence}

Seyit Uyar

E-mail: seyituyar79@hotmail.com

Funding sources

None declared

Conflict of interest

None declared

Received on March 9, 2017

Reviewed on April 17, 2017

Accepted on June 3, 2017

\section{Abstract}

Background. Patients with systemic lupus erythematosus (SLE) have an increased risk of thrombotic events. Platelets become more active and they enlarge to release proteins from alpha granules for aggregation during the plaque formation period. Beta-thromboglobulin is one of the proteins released from alpha-granules when platelets are activated and used as a marker of platelet activation in vivo.

Objectives. The aim of this study was to evaluate the plasma levels of beta-thromoglobulin and mean platelet volume as markers of the presence of platelet activation in systemic lupus erythematosus patients compared with healthy controls.

Material and methods. Thirty-seven SLE patients with a mean disease duration of 4.96 years and without any organ involvement as well as 30 healthy volunteers were included in the study. All patients were in remission of SLE.

Results. Themean beta-thromboglobulin level was $97.36 \pm 55.8 \mathrm{ng} / \mathrm{mL}$ intheSLEgroup and $72.67 \pm 33.5 \mathrm{ng} / \mathrm{mL}$ in the control group $(p=0.029$. The mean platelet volume level was $8.27 \pm 1.68 \mathrm{fL}$ in the SLE group and $9.16 \pm 1.52 \mathrm{fL}(p=0.031)$ in the controls.

Conclusions. Elevated beta-thromboglobulin levels in systemic lupus erythematosus patients may be associated with platelet activation in the early stages of disease, whereas lower mean platelet volume levels in the same population may be due to the effects of hydroxychloroquine and the inactivity of SLE.

Key words: systemic lupus erythematosus, mean platelet volume, $\beta$-thromboglobulin

DOI

10.17219/acem/74389

Copyright

Copyright by Author(s)

This is an article distributed under the terms of the

Creative Commons Attribution Non-Commercial License

(http://creativecommons.org/licenses/by-nc-nd/4.0/) 


\section{Introduction}

Systemic lupus erythematosus (SLE) is an autoimmune disease with an increased risk of thrombosis. ${ }^{1}$ Thrombotic events are becoming more of a factor on morbidity and mortality than inflammation due to developing effective anti-inflammatory treatments. ${ }^{2}$ For these reasons, SLE patients experience thrombotic events at younger ages than the general population. ${ }^{3}$ According to a study by Mok et al., women with SLE are hospitalized with myocardial infarction and stroke almost 9 times more often than the normal population. ${ }^{4}$ Longer disease duration, older age at SLE diagnosis, disease activity, smoking, and the presence of antiphospholipid antibodies have all been established as risk factors for thrombosis in SLE patients. ${ }^{5}$ Several blood parameters have been proposed to predict the occurrence of thrombosis, including the presence of lupus anticoagulant, anticardiolipin antibodies, and anti-2-glycoprotein I antibodies. However, these well-known risk factors do not explain thrombosis formation exactly. For example, approximately $40 \%$ of SLE patients with thrombosis tested negative for antiphospholipid antibodies in 2005. ${ }^{1}$ Therefore, better predictors of thrombosis are needed for the early prevention of this potentially fatal disease complication.

The activation and aggregation of platelets during hemostasis is essential for plug formation and for preventing excessive blood loss. However, if this process occurs in an uncontrolled manner it may lead to thrombotic events. During the formation of plaque, platelets become more active and they enlarge to release proteins from alpha granules for aggregation. Platelet factor 4 and $\beta$-thromboglobulin ( $\beta$-TG) are proteins released from alpha-granules when platelets become activated and can be used as a marker of platelet activation in vivo. ${ }^{6}$ An elevation in mean platelet volume (MPV) may also be an indicator for platelet activation, as demonstrated in studies of many different diseases. ${ }^{7-11}$ There have also been many studies investigating $\beta$-TG levels in different autoimmune diseases. ${ }^{12-15}$ A review of the literature, however, shows that there are few studies evaluating $\beta$-TG levels and MPV levels in SLE patients as a platelet activator. ${ }^{16-19}$

Many studies suggest that there is persistent platelet activation in SLE patients. ${ }^{16,20,21}$ In this study, we aimed to show platelet activation in the early stages of the disease and during the remission period in SLE patients by evaluating $\beta$-TG and MPV levels and comparing them with healthy controls. We can hypothesize that there is a risk of thrombosis in the early stages of SLE.

\section{Material and methods}

\section{Study design}

This study was designed as a prospective, cross-sectional clinical trial with a control group. It was performed in the outpatient clinics of the internal medicine and rheumatology departments of Haydarpasa Numune Training and
Research Hospital. The study protocol was performed in accordance with the Declaration of Helsinki and approved by the ethic committees of our hospital. Written informed consent was obtained from each participant before the commencement of the study.

Thirty-seven patients who were followed in the outpatient clinics of the rheumatology and internal medicine departments of our hospital with a diagnosis of SLE as well as 30 healthy volunteers without any systemic disease were included in the study. All patients were in the remission period of SLE and were not on any medication apart from hydroxychloroquine phosphate. The diagnosis of SLE was based on the diagnostic criteria revised by the American Rheumatism Association in 1987.22 Patients were excluded from the study if they had any disease affecting platelet function, diabetes mellitus, renal failure, liver failure, any kind of malignancy, peripheral vascular disease, any thrombotic events, cerebrovascular disease, chronic thyroid disease, hypertension, coronary artery disease, congestive heart failure, idiopathic thrombocytopenic purpura, iron deficiency anemia, or pregnancy, or if they were using any medications which affect platelet functions (for example, aspirin, clopidogrel, non-steroidal anti-inflammatory drugs, anti-coagulants, or corticosteroids) within the previous 15 days, their body mass index (BMI) was $\geq 30$, or they had active SLE. None of the patients had kidney involvement of SLE. For each individual participating in the study, a questionnaire consisting of physical examination and medical history was completed.

After the approval of informed consent from all patients, the patients were physically examined by an internist and checked for inclusion and exclusion criteria. All patients had no organ involvement according to ACR criteria and none of them had any active complaints. All patients were using only hydroxychloroquine phosphate for SLE. ${ }^{23}$ None of the patients were receiving corticosteroids and/or immune suppressant agents. After $12 \mathrm{~h}$ of fasting and half an hour of rest, venous blood samples were taken for biochemical tests between 8:00 and 8:30 am. The total cholesterol, low-density lipoprotein cholesterol (LDL-C), highdensity lipoprotein cholesterol (HDL-C), and triglyceride levels, the sedimentation rate, and C-reactive protein levels of all patients were measured in the Biochemistry Laboratory with "Beckman Coulter" device (Beckman Coulter Diagnostics, Fullerton, USA).

\section{Measurement of MPV and $\beta$-TG}

The measurement of MPV and $\beta$-TG levels was performed in the Ozel Gelisim Tip Laboratory (Istanbul, Turkey). Blood samples were taken as stated above and sodium citrate was used for the anticoagulation of the blood samples taken for MPV and $\beta$-TG. All samples were meticulously studied within half an hour. The first $2 \mathrm{~mL}$ of blood was drained out and the remaining amount was put into a special tube containing a mixture for $\beta$-TG level 
platelet markers. The tubes were kept for $15 \mathrm{~min}$ in ice water and were then centrifuged at $2-8^{\circ} \mathrm{C}$ at the 2,500 cycle for $20 \mathrm{~min}$. The middle $1 / 3$ part of the plasma of the centrifuged blood was isolated from the rest with a pipette. The samples were stored at $-20^{\circ} \mathrm{C}$ and $\beta$-TG levels were measured with an ELISA kit for human beta thromboglobulin (Elabscience Biotechnology, Wuhan, China).

\section{Statistical analysis}

Statistical analysis was performed using v. 16.0 of SPSS (Statistical Package for Social Sciences) for Windows (SPSS Inc., Chicago, USA). Besides descriptive statistical methods (mean, median, standard deviation, and range), Student's t-test was used for the comparison of the parameters showing normal distribution. The $\chi^{2}$ test or Fisher's exact test was used to compare qualitative data. The distribution of the continuous variables was tested with the OneSample Kolmogorov-Smirnov Test. A p-value of $<0.05$ was considered significant.

\section{Results}

Thirty-seven patients (SLE group) and 30 healthy volunteers (control group) who were similar to the patient group in terms of age and gender distribution were included in the study. The SLE group consisted of 34 females and 3 males, and the mean age was $35.78 \pm 8.93$ years. The healthy control group consisted of 26 females and 4 males and the mean age was $34.76 \pm 9.84$ years. There was no significant difference between the groups in terms of age or gender distribution $(p>0.05)$. The mean age of diagnosis was $33.05 \pm 9.05$ years and the mean disease duration was $4.96 \pm 4.97$ years for SLE patients (Table 1 ).

The demographic characteristics, cholesterol levels, acute phase reactants, and complement levels of the SLE and control groups are presented in Table 1. There was no statistical difference between the groups with respect to these parameters ( $\mathrm{p}>0.05)$.

The number of patients who smoked in the SLE group was 13 and in the control group it was 9. The difference between the groups was not statistically significant ( $\mathrm{p}=0.656$ ) (Table 1). There was no difference between the groups in terms of platelet count $(\mathrm{p}>0.05)$. The mean $\beta$-TG levels in the SLE group was $97.36 \pm 55.85 \mathrm{ng} / \mathrm{mL}$ and $72.67 \pm 33.55 \mathrm{ng} / \mathrm{mL}$ in the healthy controls. The difference between the groups in terms of $\beta$-TG levels was found to be significant $(\mathrm{p}=0.029)$ (Table 2$)$.

The mean MPV value of the SLE group was $8.27 \pm 1.68 \mathrm{fL}$ and in the control group it was $9.16 \pm 1.52 \mathrm{fL}$. The difference between these groups in terms of MPV levels was significant $(\mathrm{p}=0.031)$ (Table 2$)$.

Table 1. Demographic characteristics, cholesterol levels, acute phase reactants, and complement levels of the groups

\begin{tabular}{|c|c|c|c|}
\hline Parameters & SLE patients $(n=37)$ & Healthy controls $(n=30)$ & $\mathrm{p}$-value \\
\hline Age [years], mean $\pm \mathrm{SD}$ & $35.78 \pm 8.93$ & $34.76 \pm 9.84$ & $0.66^{*}$ \\
\hline Female, $n$ & 34 & 26 & $0.692^{* *}$ \\
\hline Smoking status, $\mathrm{n}$ & 13 & 9 & $0.656^{* *}$ \\
\hline Total cholesterol $[\mathrm{mg} / \mathrm{dL}]$, mean $\pm \mathrm{SD}$ & $178.46 \pm 38.41$ & $185.0 \pm 31.21$ & $0.455^{*}$ \\
\hline High-density lipoproteins [mg/dL], mean \pm SD & $59.37 \pm 17.33$ & $53.96 \pm 9.02$ & $0.106^{*}$ \\
\hline Low-density lipoproteins [mg/dL], mean \pm SD & $95.62 \pm 32.26$ & $110.33 \pm 27.91$ & $0.053^{*}$ \\
\hline Triglycerides $[\mathrm{mg} / \mathrm{dL}]$, mean $\pm \mathrm{SD}$ & $117.81 \pm 63.61$ & $101.20 \pm 50.21$ & $0.248^{*}$ \\
\hline Age of diagnosis [years], mean $\pm \mathrm{SD}$ & $33.05 \pm 9.05$ & - & - \\
\hline Disease duration [years], mean $\pm \mathrm{SD}$ & $4.96 \pm 4.97$ & - & - \\
\hline Sedimentation rate $[\mathrm{mm} / \mathrm{h}]$, median (min-max) & $11(3-73)$ & - & - \\
\hline C-reactive protein [mg/dL], median (min-max) & $0.10(0.06-2.7)$ & - & - \\
\hline White blood cell $\left[\times 10^{3} / \mathrm{LL}\right]$, mean $\pm \mathrm{SD}$ & $6.84 \pm 2.35$ & - & - \\
\hline Complement C3 [mg/dL], mean \pm SD & $87.46 \pm 2.26$ & - & - \\
\hline Complement C4 [mg/dL], mean \pm SD & $16.69 \pm 6.51$ & - & - \\
\hline
\end{tabular}

* Student's t-test; ** $\chi^{2}$ test; SLE - systemic lupus erythematosus.

Table 2. Comparison of the groups in terms of $\beta$-thromboglobulin, MPV, and platelet count levels

\begin{tabular}{|l|c|c|}
\multicolumn{1}{|c|}{ Parameters } & SLE patients $(\mathrm{n}=37)$ & Healthy controls $(\mathrm{n}=30)$ \\
\hline$\beta$-thromboglobulin $[\mathrm{ng} / \mathrm{mL}]$, mean $\pm \mathrm{SD}$ & $97.36 \pm 55.85$ & $72.67 \pm 33.55$ \\
\hline MPV $[\mathrm{fL}]$, mean \pm SD & $8.27 \pm 1.68$ & 0.029 \\
\hline Platelet count, mean \pm SD & $211,406.25 \pm 81,476.63$ & 0.031 \\
\hline
\end{tabular}

* Student's t-test; SLE - systemic lupus erythematosus; MPV - mean platelet volume. 


\section{Discussion}

The $\beta$-TG levels were significantly higher and the MPV levels were significantly lower in the SLE group than in the healthy controls in this study. The SLE group and the healthy controls were similar demographically and in terms of cardiac risk factors (e.g., age, sex, smoking, and lipid levels). All patients were in remission for SLE because the sedimentation rate and the C-reactive protein, white blood cell, and complement levels of patients were within normal range. The mean age of diagnosis was 33.05 years and the mean disease duration of the patients was 4.96 years. Due to the increased levels of $\beta-T G$, it can be assumed that platelet activation occurs in the early course of the disease and during the remission period, according to our findings. Ekdahl et al. studied $\beta$-TG, phosphorylated fibrinogen, and activated factor IX-antithrombin complexes in SLE patients with and without deep vein thrombosis (DVT) and compared these levels to patients with DVT and to healthy individuals. They reported that high levels of $\beta$-TG and phosphorylated fibrinogen in the samples from patients with SLE were strongly associated with thrombosis. $\beta$-TG levels of SLE patients without thrombosis were also higher than the normal range in their study. ${ }^{16}$ When we evaluate our results together with the Ekdahl study, we may conclude that SLE patients may be prone to thrombosis in the early course of the disease. This hypothesis is compatible with a study done by Chang et al. ${ }^{24}$ They concluded that thrombovascular events occur throughout the course of lupus with the highest risk in the first year after diagnosis. ${ }^{24}$

$\beta$-TG levels were higher and MPV values were significantly lower in SLE patients, though these results may seem contradictory. There are a few studies investigating MPV levels in SLE patients and their results are also contradictory. In one of the earliest laboratory investigations, Turner-Stokes et al. reported that the platelet count and volume were significantly lower in SLE patients according to 2 different hematology analyzers. ${ }^{18}$ In a recent study, MPV levels were found to be lower in adult patients with active SLE as compared to inactive patients. In that study, there was no healthy control group and MPV values were $7.16 \mathrm{fL}$ and $8.16 \mathrm{fL}$ in active and inactive SLE patients, respectively. ${ }^{19}$ Safak et al. also found that the MPV levels in active SLE patients were significantly lower than in SLE patients in remission and in healthy controls, which is consistent with our results. ${ }^{25}$ Lower MPV levels correlated with active inflammatory states in that study. In another study, Yolbas et al. reported that MPV levels were higher (but not statistically significantly higher) in SLE patients than in healthy controls. ${ }^{26}$ Although the findings of this study seem to be the opposite of our findings, the mean SLEDAI score of SLE patients was 12.1 in that study. Our study population had no complaints of SLE and were in remission; this may be the reason for the lower MPV levels in this study.
This discordance of MPV levels between studies may be explained by the different methodologies and designs of the studies. The blood sample anticoagulation type (ethylenediaminetetraacetic acid [EDTA] used as an anticoagulant), a delay in the processing of blood samples, anti-inflammatory therapies, and smoking are a few such differences. We processed samples quickly (in under $1 \mathrm{~h}$ ) and used tubes with sodium citrate to minimize technical mistakes. We did not exclude smokers, but there was no difference in the smoking status between patients and controls in our study.

All patients in our study were taking only hydroxychloroquine for SLE. Although not fully understood, hydroxychloroquine may have potential anti-thrombotic effects through several mechanisms. ${ }^{27}$ The inhibition of platelet aggregation, the inhibition of the intravascular aggregation of red blood cells, and the inhibition of the binding of antiphospholipid antibodies to phospholipid surfaces are the mechanisms of the potential anti-thrombotic actions of hydroxychloroquine. ${ }^{27}$ In the literature, there is not a single study precisely investigating the effects of drugs, including hydroxycholoroquine, used for SLE on the platelets. ${ }^{28}$ The effect of hydroxychloroquine may be another reason for the lower MPV levels in our study, but this contrasts with higher $\beta$-TG levels in same group. Further studies that include more patients are needed for more precise elucidation of this issue.

We have included in the study patients with no organ involvement and whose disease duration was less than 10 years. We may include some of the exclusion criteria and the small sample size as limitations of this study.

\section{Conclusions}

Elevated $\beta$-TG levels in SLE patients who were in the early stages of disease and in remission may support the hypothesis that there is platelet activation throughout the course of SLE. In contrast, lower MPV levels in the same population may be associated with the hydroxychloroquine effect and the inactivity of SLE. Clinicians should also watch out for thrombosis in the early stages of SLE, even if the patients have no organ involvement. However, further prospective, controlled studies with larger populations are needed in this area.

\section{References}

1. Afeltra A, Vadacca M, Conti L, et al. Thrombosis in systemic lupus erythematosus: Congenital and acquired risk factors. Arthritis Rheum. 2005;53(3):452-459.

2. Nowak B, Szmyrka-Kaczmarek M, Durazińska A, et al. Anti-ox-LDL antibodies and anti-ox-LDL-B2GPI antibodies in patients with systemic lupus erythematosus. Adv Clin Exp Med. 2012;21(3):331-335.

3. Cervera R, Khamashta MA, Font J, et al. Morbidity and mortality in systemic lupus erythematosus during a 10-year period: A comparison of early and late manifestations in a cohort of 1,000 patients. Medicine (Baltimore). 2003;82(5):299-308. 
4. Mok CC, Tang SS, To CH, et al. Incidence and risk factors of thromboembolism in systemic lupus erythematosus: A comparison of three ethnic groups. Arthritis Rheum. 2005;52(9):2774-2782.

5. Kaiser R, Li Y, Chang M, et al. Genetic risk factors for thrombosis in systemic lupus erythematosus. J Rheumatol. 2012;39(8):1603-1610.

6. Kaplan KL, Owen J. Plasma levels of beta-thromboglobulin and platelet factor 4 as indices of platelet activation in vivo. Blood. 1981;57(2): 199-202.

7. Endler G, Klimesch A, Sunder-Plassmann H, et al. Mean platelet volume is an independent risk factor for myocardial infarction but for coronary artery disease. Br J Haematol. 2002;117(2):399-404.

8. Yazici S, Yazici M, Erer B, et al. The platelet indices in patients with rheumatoid arthritis: Mean platelet volume reflects disease activity. Platelets. 2010;21(2):122-125.

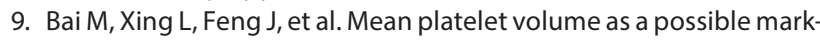
er for monitoring the disease activity in ulcerative colitis. Int J Lab Hematol. 2016. https://doi.org/10.1111/ijlh.12495

10. Pyo JS, Sohn JH, Kang G. Diagnostic and prognostic roles of the mean platelet volume in malignant tumors: A systematic review and metaanalysis. Platelets. 2016;9:1-7.

11. Lippi G, Danese E, Montagnana M, et al. Mean platelet volume is significantly associated with serum levels of thyroid-stimulating hormone in a cohort of older euthyroid subjects. Endocr Res. 2015;40(4): 227-230.

12. Karatoprak C, Uyar S, Abanonu GB, et al. The levels of $\beta$-thromboglobulin in femalerheumatoid arthritis patients as activation criteria. Rheumatol Int. 2013;33(5):1229-1232.

13. Abanonu GB, Daskin A, Akdogan MF, et al. Mean platelet volume and $\beta$-thromboglobulin levels in familial Mediterranean fever: Effect of colchicine use? Eur J Intern Med. 2012;23(7):661-664.

14. Yamamoto T, Chikugo T, Tanaka Y. Elevated plasma levels of betathromboglobulin and platelet factor 4 in patients with rheumatic disorders and cutaneous vasculitis. Clin Rheumatol. 2002;21(6):501-504

15. Vrij AA, Rijken J, Van Wersch JW, et al. Platelet factor 4 and betathromboglobulin in inflammatory bowel disease and giant cell arteritis. Eur J Clin Invest. 2000;30(3):188-194.

16. Ekdahl KN, Bengtsson AA, Andersson J, et al. Thrombotic disease in systemic lupus erythematosus is associated with a maintained systemic platelet activation. Br J Haematol. 2004;125(1):74-78.
17. Tam LS, Fan B, Li EK, et al. Patients with systemic lupus erythematosus show increased platelet activation and endothelial dysfunction induced by acute hyperhomocysteinemia. J Rheumatol. 2003;30(7): 1479-1484.

18. Turner-Stokes $L$, Jones D, Patterson KG, et al. Measurement of haematological indices of chronic rheumatic disease with two newer generation automated systems, the $\mathrm{H} 1$ and $\mathrm{H} 6000$ (Technicon). Ann Rheum Dis. 1991;50:583-587.

19. Delgado-García G, Galarza-Delgado DÁ, Colunga-Pedraza I, et al. Mean platelet volume is decreased in adults with active lupus disease. Rev Bras Reumatol. 2016;56(6):504-508.

20. Joseph JE, Harrison P, Mackie IJ, et al. Increased circulating platelet-leucocyte complexes and platelet activation in patients with antiphospholipid syndrome, systemic lupus erythematosus and rheumatoid arthritis. Br J Haematol. 2001;115(2):451-459.

21. Bäck J, Lood C, Bengtsson AA, et al. Contact activation products are new potential biomarkers to evaluate the risk of thrombotic events in systemic lupus erythematosus. Arthritis Res Ther. 2013. doi: 10.1186/ ar4399

22. Tan E, Cohen A, Fries J, et al. The 1982 revised criteria for the classification of systemic lupus erythematosis. Arthritis Rheum. 1982;25: 1271-1277.

23. Hochberg MC. Updating the American College of Rheumatology revised criteria for the classification of systemic lupus erythematosus. Arthritis Rheum. 1997;40(9):1725.

24. Chang ER, Pineau CA, Bernatsky S, et al. Risk for incident arterial or venous vascular events varies over the course of systemic lupus erythematosus. J Rheumatol. 2006;33(9):1780-1784.

25. Safak S, Uslu AU, Serdal K, et al. Association between mean platelet volume levels and inflammation in SLE patients presented with arthritis. Afr Health Sci. 2014;14(4):919-924.

26. Yolbas S, Yildirim A, Gozel N, et al. Hematological indices may be useful in the diagnosis of systemic lupus erythematosus and in determining disease activity in Behçet's disease. Med Princ Pract. 2016; 25(6):510-516.

27. Belizna C. Hydroxychloroquine as an anti-thrombotic in antiphospholipid syndrome. Autoimmun Rev. 2015;14(4):358-362.

28. Gasparyan AY, Ayvazyan L, Pretorius E, et al. Platelets in rheumatic diseases: Friend or foe? Curr Pharm Des. 2014;20(4):552-566. 